PROCEEDINGS OF THE

AMERICAN MATHEMATICAL SOCIETY

Volume 125, Number 4, April 1997, Pages 1193-1196

S 0002-9939(97)03668-X

\title{
HYPERSURFACES IN A SPHERE WITH CONSTANT MEAN CURVATURE
}

\author{
ZHONG HUA HOU \\ (Communicated by Peter Li)
}

\begin{abstract}
Let $M^{n}$ be a closed hypersurface of constant mean curvature immersed in the unit sphere $S^{n+1}$. Denote by $S$ the square of the length of its second fundamental form. If $S<2 \sqrt{n-1}, M$ is a small hypersphere in $S^{n+1}$. We also characterize all $M^{n}$ with $S=2 \sqrt{n-1}$.
\end{abstract}

\section{INTRODUCTION}

Let $M^{n}$ be a closed submanifold with parallel mean curvature vector field immersed in the unit sphere $S^{n+p}$. Denote by $H$ the length of the mean curvature vector field and by $S$ the square of the length of the second fundamental form of $M^{n}$. It is important to characterize those $M$ immersed as $n$-spheres in $S^{n+p}$ by $H$ and $S$.

When $M$ is minimal, J. Simons [9] obtained a pinching constant $n /(2-1 / p)$ of $S$ and Chern-do Carmo-Kobayashi [3] showed that it is sharp and characterized all $M$ with $S=n /(2-1 / p)$. M. Okumura [6,7] first discussed the general case and gave a pinching constant of $S$, but it is not sharp. Recently the sharp ones were obtained by H. Alencar-M. do Carmo [1] for $p=1$, W. Santos [8] for $p>1$ and H. W. Xu [11] for $p \geq 1$ respectively. But all of them were expressed by the mean curvature $H$. S. T. Yau [12] obtained a pinching constant for $p>1$ which depended only on $n$ and $p$. H. W. Xu [10] improved Yau's result, but far from sharpness.

In the present paper, we shall give a pinching constant for $p=1$ which depends only on $n$ and show the sharpness of it. More precisely, we want to prove the following theorems:

Theorem A. Let $M^{n}$ be a hypersurface of constant mean curvature immersed in $S^{n+1}$ with constant length of the second fundamental form. Then:

(1) If $S<2 \sqrt{n-1}, M^{n}$ is locally a piece of small hypersphere $S^{n}(r)$ of radius $r=\sqrt{n /(n+S)}$.

(2) If $S=2 \sqrt{n-1}, M$ is locally a piece of either $S^{n}\left(r_{0}\right)$ or $S^{1}(r) \times S^{n-1}(s)$ where $r_{0}^{2}=n /(n+2 \sqrt{n-1}), r^{2}=1 /(\sqrt{n-1}+1)$ and $s^{2}=\sqrt{n-1} /(\sqrt{n-1}+1)$.

Theorem $\mathbf{A}^{\prime}$. Let $M^{n}$ be a closed hypersurface of constant mean curvature immersed in $S^{n+1}$. Then:

(1) If $S<2 \sqrt{n-1}, M^{n}$ is a small hypersphere $S^{n}(r)$ of radius $r=\sqrt{n /(n+S)}$.

Received by the editors July 27, 1995.

1991 Mathematics Subject Classification. Primary 53C42, 53A10.

(C)1997 American Mathematical Society 
(2) If $S=2 \sqrt{n-1}, M$ is either a small hypersphere $S^{n}\left(r_{0}\right)$ or a $H(r)$-torus $S^{1}(r) \times S^{n-1}(s)$, where $r_{0}, r$ and $s$ are taken as before.

The author would like to express deep gratitude to Professor S. Tanno for his continuous encouragement and patient advice.

\section{Proof of the TheOREMS}

Let $M$ be a closed hypersurface immersed in the unit sphere $S^{n+1}$. Take a local orthonormal coframe field $\left\{\omega_{i}\right\}_{i=1}^{n}$ on $M$. Then the second fundamental form can be expressed as $L=\left(h_{i j}\right)_{n \times n}$. The mean curvature $H$ and the square of the length of the second fundamental form $S$ are defined by $H=\frac{1}{n} \sum_{(i)} h_{i i}, S=\sum_{(i, j)}\left(h_{i j}\right)^{2}$.

From now on, we shall always use $i, j, k, \ldots$ for indices running from 1 to $n$.

Denote the covariant differentials of $\left\{h_{i j}\right\}$ by $\left\{h_{i j k}\right\}$ and $\left\{h_{i j k l}\right\}$. Then the Laplacian of $h_{i j}$ is defined by $\Delta h_{i j}=\sum_{(k)} h_{i j k k}$. It follows that

$$
\sum_{(i, j)} h_{i j} \Delta h_{i j}=n S+n H f-n^{2} H^{2}-S^{2},
$$

where $f=\operatorname{Tr} L^{3}$ (cf. e.g. [2] or [7]).

M. Okumura [7] established the following lemma (see also [1] or [11]).

Lemma. Let $\left\{a_{i}\right\}_{i=1}^{n}$ be a set of real numbers satisfying $\sum_{(i)} a_{i}=0, \sum_{(i)} a_{i}^{2}=t^{2}$, where $t \geq 0$. Then we have

$$
-\frac{n-2}{\sqrt{n(n-1)}} t^{3} \leq \sum_{(i)} a_{i}^{3} \leq \frac{n-2}{\sqrt{n(n-1)}} t^{3}
$$

and equalities hold if and only if at least $(n-1)$ of the $a_{i}$ 's are equal to one another.

Suppose that $\lambda_{1}, \lambda_{2}, \ldots, \lambda_{n}$ are the principal curvatures of $M$. Then we have

$$
n H=\sum_{(i)} \lambda_{i}, \quad S=\sum_{(i)} \lambda_{i}^{2}, \quad f=\sum_{(i)} \lambda_{i}^{3} .
$$

Set $\widetilde{S}=S-n H^{2}, \tilde{f}=f-3 H S+2 n H^{3}$ and $\tilde{\lambda}_{i}=\lambda_{i}-H(1 \leq i \leq n)$. Then (3) changes into

$$
0=\sum_{(i)} \tilde{\lambda}_{i}, \quad \widetilde{S}=\sum_{(i)} \tilde{\lambda}_{i}^{2}, \quad \tilde{f}=\sum_{(i)} \tilde{\lambda}_{i}^{3}
$$

By applying Okumura's Lemma to $\tilde{f}$ in (4), we have

$$
\tilde{f} \geq-\frac{n-2}{\sqrt{n(n-1)}} \widetilde{S} \sqrt{\widetilde{S}} \Longleftrightarrow f \geq 3 H S-2 n H^{3}-\frac{n-2}{\sqrt{n(n-1)}} \widetilde{S} \sqrt{\widetilde{S}}
$$

Substituting this into (1), we have

$$
\sum_{(i, j)} h_{i j} \Delta h_{i j} \geq \widetilde{S}\left\{n-\left(\widetilde{S}-n H^{2}\right)-(n-2) H \sqrt{\frac{n}{n-1} \widetilde{S}}\right\} .
$$

Consider the quadratic form $Q(u, t)=u^{2}-\frac{n-2}{\sqrt{n-1}} u t-t^{2}$. By the orthogonal transformation

$$
\left\{\begin{array}{l}
\tilde{u}=\frac{1}{\sqrt{2 n}}\{(1+\sqrt{n-1}) u+(1-\sqrt{n-1}) t\} \\
\tilde{t}=\frac{1}{\sqrt{2 n}}\{(\sqrt{n-1}-1) u+(\sqrt{n-1}+1) t\}
\end{array}\right.
$$


$Q(u, t)$ turns into $Q(u, t)=\frac{n}{2 \sqrt{n-1}}\left(\tilde{u}^{2}-\tilde{t}^{2}\right)$, where $\tilde{u}^{2}+\tilde{t}^{2}=u^{2}+t^{2}=S$.

Take $t=\sqrt{\widetilde{S}}$ and $u=\sqrt{n} H$ in $Q(u, t)$, and substitute it into (5). We can see

$$
\sum_{(i, j)} h_{i j} \Delta h_{i j} \geq \widetilde{S}\left(n-\frac{n}{2 \sqrt{n-1}} S+\frac{n}{\sqrt{n-1}} \tilde{u}^{2}\right) \geq \widetilde{S}\left(n-\frac{n}{2 \sqrt{n-1}} S\right) .
$$

Therefore we have

$$
\frac{1}{2} \Delta S=\sum_{(i, j, k)} h_{i j k}^{2}+\sum_{(i, j)} h_{i j} \Delta h_{i j} \geq \widetilde{S}\left(n-\frac{n}{2 \sqrt{n-1}} S\right) .
$$

Theorem A. Let $M^{n}$ be a hypersurface of constant mean curvature immersed in $S^{n+1}$ with constant length of the second fundamental form. Then:

(1) If $S<2 \sqrt{n-1}, M$ is locally a piece of a small hypersphere $S^{n}(r)$ in $S^{n+1}$, where $r=\sqrt{n /(n+S)}$.

(2) If $S=2 \sqrt{n-1}, M$ is locally a piece of either $S^{n}\left(r_{0}\right)$ or $S^{1}(r) \times S^{n-1}(s)$, where $r_{0}^{2}=n /(n+2 \sqrt{n-1}), r^{2}=1 /(\sqrt{n-1}+1)$ and $s^{2}=\sqrt{n-1} /(\sqrt{n-1}+1)$.

Proof. Since $S$ is constant, the left-hand side of (7) is zero. When $S \leq 2 \sqrt{n-1}$, we have

$$
\widetilde{S}\left(n-\frac{n}{2 \sqrt{n-1}} S\right)=0, \quad h_{i j k}=0, \quad 1 \leq i, j, k \leq n .
$$

If $S<2 \sqrt{n-1}$, we have $\widetilde{S}=0$, which means that $M$ is totally umbilical and hence is locally a piece of hypersphere $S^{n}(r)$ where $r=\sqrt{n /(n+S)}$.

Suppose $S=2 \sqrt{n-1}$. Then all of the inequalities in (5)-(7) become equal ones. Okumura's Lemma implies that at least $(n-1)$ of $\lambda_{i}$ 's are equal to one another. When $\lambda_{1}=\lambda_{2}=\cdots=\lambda_{n}, M$ is totally umbilical and hence is locally a piece of hypersphere $S^{n}(r)$ where $r^{2}=n /(n+2 \sqrt{n-1})$. When $M$ is not totally umbilical, there are exactly $(n-1)$ of $\lambda_{i}$ 's that are equal to one another. The same arguments as those developed by Chern-do Carmo-Kobayashi (see [3], p. 68) show that $M$ is locally a piece of $S^{1}(r) \times S^{n-1}(s)$ in $S^{n+1}$. To determine the radii $r$ and $s$, we refer to the examples of K. Nomizu and B. Smyth [5], from which we have

$$
H=-\frac{1}{n}\left(\frac{s}{r}\right)+\frac{n-1}{n}\left(\frac{r}{s}\right), \quad S=\left(\frac{s}{r}\right)^{2}+(n-1)\left(\frac{r}{s}\right)^{2} .
$$

It is easy to see that

$$
\left(\frac{s}{r}\right)^{2}+(n-1)\left(\frac{r}{s}\right)^{2} \geq 2 \sqrt{n-1}
$$

and equality holds if and only if $\left(\frac{s}{r}\right)^{2}=\sqrt{n-1}$. Therefore we have $r^{2}=\frac{1}{\sqrt{n-1}+1}$ and $s^{2}=\frac{\sqrt{n-1}}{\sqrt{n-1}+1}$.

When $M$ is closed, the integral of the left-hand side of (7) on $M$ is equal to zero, and so is that of the right-hand side. After the same deduction as in the proof of Theorem A, we can obtain the following:

Theorem A'. Suppose $M$ is a closed hypersurface of constant mean curvature immersed in $S^{n+1}$. Then:

(1) If $S<2 \sqrt{n-1}, M$ is a small hypersphere $S^{n}(r)$, where $r=\sqrt{n /(n+S)}$.

(2) If $S=2 \sqrt{n-1}, M$ is either a small hypersphere $S^{n}\left(r_{0}\right)$ or $S^{1}(r) \times S^{n-1}(s)$, where $r_{0}, r$ and $s$ are taken as in Theorem $A$. 
We can show an application of Theorem A'. H. W. Xu [10] proved the following:

Proposition $(\mathrm{Xu})$. Let $M^{n}$ be an $n$-dimensional compact submanifold with parallel mean curvature vector field in $S^{n+p}$ and $p>1$. If

$$
S \leq \min \left\{\frac{2 n}{1+\sqrt{n}}, \frac{n}{2-(p-1)^{-1}}\right\}
$$

and the Gauss mapping of $M$ is relatively affine, then $M^{n}$ is a standard hypersphere in a totally geodesic $S^{n+1}$ of $S^{n+p}$.

By Theorem $\mathrm{A}^{\prime}$, we can remove the assumption that the Gauss mapping is relatively affine. Namely we can obtain the following

Corollary. Let $M^{n}$ be an n-dimensional compact submanifold with parallel mean curvature vector field in $S^{n+p}$ and $p>1$. If

$$
S \leq \min \left\{\frac{2 n}{1+\sqrt{n}}, \frac{n}{2-(p-1)^{-1}}\right\}
$$

then $M^{n}$ is a standard hypersphere in a totally geodesic $S^{n+1}$ of $S^{n+p}$.

Proof. It is easy to check that $(\sqrt{n}+1) / n>1 / \sqrt{n-1}$. Therefore we have

$$
\sqrt{n-1}>\frac{n}{\sqrt{n}+1} \Longleftrightarrow 2 \sqrt{n-1}>\frac{2 n}{\sqrt{n}+1} \geq S .
$$

\section{REFERENCES}

[1] H. Alencar and M. P. do Carmo, Hypersurfaces with constant mean curvature in spheres, Proc. Amer. Math. Soc. 120 (1994), pp. 1223-1229. MR 94f:53108

[2] S. Y. Cheng and S. T. Yau, Hypersurfaces with constant scalar curvature, Math. Ann. 225 (1977), pp. 195-204. MR 55:4045

[3] S. S. Chern, M. do Carmo and S. Kobayashi, Minimal submanifolds of a sphere with second fundamental form of constant length, Functional Analysis and Related Fields (Proc. Conf. for M. Stone), Springer-Verlag, New York (1970), pp. 59-75. MR 42:8424

[4] H. B. Lawson, Jr., Local rigidity theorems for minimal hypersurfaces, Ann. of Math. (2) 89 (1969), pp. 187-197. MR 38:6505

[5] K. Nomizu and B. Smyth, A formula of Simons' type and hypersurfaces of constant mean curvature, J. Diff. Geom. 3 (1969), pp. 367-378. MR 42:1018

[6] M. Okumura, Submanifolds and a pinching problem on the second fundamental tensors, Trans. Amer. Math. Soc. 178 (1973), pp. 285-291. MR 47:5793

[7] _ Hypersurfaces and a pinching problem on the second fundamental tensor, Amer. J. Math. 96 (1974), pp. 207-213. MR 50:5701

[8] W. Santos, Submanifolds with parallel mean curvature vector spheres, Tôhoku Math. J. 46 (1994), pp. 403-415. MR 95f:53109

[9] J. Simons, Minimal varieties in Riemannian manifolds, Ann. of Math. (2) 88 (1968), pp. 62-105. MR 38:1617

[10] H. W. Xu, A pinching constant of Simon's type and isometric immersion, Chinese Ann. of Math. Ser. A 12 (1991), No. 3, pp. 261-269. MR 92h:53077

[11] _ A rigidity theorem for submanifolds with parallel mean curvature in a sphere, Arch. Math. 61 (1993), pp. 489-496. MR 94m:53084

[12] S. T. Yau, Submanifolds with constant mean curvature II, Amer. J. Math. 97 (1975), No. 1, pp. 76-100. MR 51:6670

Department of Mathematics, Tokyo Institute of Technology, Japan

E-mail address: hou@math.titech.ac.jp

Department of Applied Mathematics, Dalian University of Technology, People's Republic of China 